\title{
PROBABILISTIC ANALYSIS OF TWO RELIABILITY MODELS OF A SINGLE-UNIT SYSTEM WITH PREVENTIVE MAINTENANCE BEYOND WARRANTY AND DEGRADATION
}

\section{ANALIZA PROBABILISTYCZNA DWÓCH MODELI NIEZAWODNOŚCI SYSTEMU JEDNOELEMENTOWEGO WYKORZYSTUJĄCYCH POJĘCIA POGWARANCYJNEJ OBSŁUGI PROFILAKTYCZNEJ ORAZ DEGRADACJI}

\begin{abstract}
This paper presents two reliability models of a single-unit system with the concept of preventive maintenance (PM) beyond warranty and degradation. In both the models, repair of any failure during warranty is cost-free to the users, provided failures are not due to the negligence of users. There is a single repairman who always remains with the system. Beyond warranty, the unit goes under PM and works as new after PM (in both models). In model-1, the unit works as new after its repair beyond warranty whereas; in model-2, the unit becomes degraded. After failure, the degraded unit is replaced by a new one. The failure time of the system follows negative exponential distribution while PM, replacement and repair time distributions are taken as arbitrary with different probability density functions. Supplementary variable technique is adopted to derive the expressions for some economic measures such as reliability, mean time to system failure (MTSF), availability and profit function. Using Abel's lemma, the behaviour of the system in steady-state has been examined. To highlight the behaviour of reliability and profit function, numerical results are considered for particular values of various parameters and repair cost. Profit comparison of both the models is also made to see the usefulness of the concept of degradation.
\end{abstract}

Keywords: probabilistic analysis, reliability, preventive maintenance, warranty, degradation.

$W$ artykule przedstawiono dwa modele niezawodności systemu jednoelementowego wykorzystujace pojęcia pogwarancyjnej obstugi profilaktycznej oraz degradacji. Oba modele zakładaja, że w okresie gwarancyjnym użytkownik nie ponosi żadnych kosztów zwiąanych z naprawa uszkodzeń, chyba że uszkodzenie powstato wskutek zaniedbania ze strony użtkownika. Obstugi sa wykonywane przez jedna ekipę remontowa, która zawsze pozostaje na stanowisku. Po upływie okresu gwarancyjnego, urządzenie podlega obstudze profilaktycznej i po jej przeprowadzeniu działa jak nowe (w obu modelach). Model 1 zaktada, że element po naprawie pogwarancyjnej działa jak nowy, natomiast w Modelu 2, element ulega degradacji. Zdegradowany element, który ulegt uszkodzeniu, zostaje wymieniony na nowy. Rozkład czasu uszkodzenia jest rozkładem wykładniczym ujemnym, a rozkłady czasu obstugi profilaktycznej, wymiany i naprawy sa traktowane jako arbitralne, o różnych funkcjach gęstości prawdopodobieństwa. Zastosowana technika dodatkowej zmiennej pozwoliła na wyprowadzenie wyrażeń dla niektórych miar ekonomicznych, takich jak niezawodność, średni czas do uszkodzenia systemu (MTSF), gotowość i funkcja zysków. Zachowanie systemu w stanie ustalonym badano z wykorzystaniem lematu Abela. Aby przedstawić zachowanie funkcji niezawodności i zysków, analizowano wyniki numeryczne dla poszczególnych wartości różnych parametrów oraz kosztów naprawy. Porównanie zyskowności badanych modeli umożliwiło weryfikację przydatności pojęcia degradacji.

Stowa kluczowe: analiza probabilistyczna, niezawodność, obshuga profilaktyczna, gwarancja, degradacja.

\section{Introduction}

In modern marketplace, warranty has its own priority in business for manufacturers to protect their benefits and to compete with other manufacturers. By using regenerating point and semi-Markov technique, various researchers including Kadyan et al. [3], Yang and Dhillon [6], Perez Ocon and Ruiz Castro [8], Philip and Cristiano [9] and Yuan and Meng [11] have studied reliability models of one or more unit systems under different sets of assumptions on failure and repair policies. When the failure rate or repair rate or both are timedependent, the system loses its Markov character and becomes nonMarkovian. By introducing one or more supplementary variables, the non-Markovian nature of the process is changed to Markovian. Firstly, Cox [1] used supplementary variable technique in analyzing non-
Markovian stochastic process. Singh et al. [10] studied a system having two units in series configuration with controller and Nailwal and Singh [7] analyzed an operating system with inspection in different weather condition by using supplementary variable technique without considering the concept of cost-free warranty. But, warranty assured the customers that the products they are buying perform satisfactorily for a particular period of time and markets the product.

Also, performing PM has become prevalent to improve the condition of the deteriorated product (or system) and reduce the cost of repairing deteriorated product. Kadyan [2] discussed reliability and profit analysis of a single-unit system with preventive maintenance without considering degradation of the unit after its repair.

However, the failed unit does not always work as new after its repair. Due to continuous usage and ageing effect, failure rate of a unit 
may increase after its repair. In such a situation, unit works with reduced capacity after its repair and so is called a degraded unit. Kumar et al. [4,5] analysed redundant systems with degradation of the unit after repair without any warranty.

In view of the above observations, here we developed two reliability models of a single-unit system with the concept of PM beyond warranty and degradation. In both the models, repair of any failure during warranty is cost-free to the users, provided failures are not due to the negligence of users such as cracked screen, accident, misuse, physical damage, damage due to liquid and unauthorized modifications etc. There is a single repairman, who always remains with the system. Beyond warranty, the unit goes under PM and works as new after PM (in both the models). In model-1, the unit works as new after its repair beyond warranty whereas; in model-2, the unit becomes degraded. In model-2, the degraded unit is replaced by a new one after its failure. The failure time of the system follows negative exponential distribution while PM, replacement and repair time distributions are taken as arbitrary with different probability density functions. Supplementary variable technique is adopted to derive the expressions for some economic measures such as reliability, MTSF, availability and profit function. Using Abel's lemma ([6] \& [9]), the behaviour of the system in steady-state has been examined. To highlight the behaviour of reliability and profit function, numerical results are also considered for particular values of various parameters and repair cost. Profit comparison of both models is made to see the usefulness of the concept of degradation.

\section{Notations}

$\lambda / \lambda_{1}$ Constant failure rate of the new unit within/beyond warranty.

$\lambda_{2}$ Constant failure rate of the degraded unit beyond warranty.

$\lambda_{m} \quad$ Transition rate with which a unit goes under PM for improvement.

$\alpha \quad$ Transition rate with which warranty of the system is completed.

$\mu(x), S(x) / \mu_{1}(x), S_{1}(x)$ Repair rate of the unit and probability density function, for the elapsed repair time $x$ within/ beyond warranty.

$\mu_{2}(y), S_{2}(y) \quad$ PM rate of the unit and probability density function, for the elapsed PM time $y$.

$\mu_{3}(z), S_{3}(z)$ Replacement rate of the failed degraded unit and probability density function, for the elapsed replacement time $z$.

$p_{0}(t) / p_{1}(t) \quad$ Probability density that at time $t$, the system is within/ beyond warranty and in good state.

$p_{i}(x, t) \quad$ Probability density that at time $t$, the system is in state $S_{i}$, $i=2,4$ and the system is under repair with elapsed repair time $x$.

$p_{3}(y, t) \quad$ Probability density that at time $t$, the system is in state $S_{3}$ and the unit is under PM with elapsed PM time $y$.

$p_{5}(t) \quad$ Probability density that at time $t$, the system is operable and in degraded state. $p_{6}(z, t)$ Probability density that at time t, the system is in state $\mathrm{S} 6$ and the failed degraded unit is under replacement with elapsed replacement time $z$.

$p(s) \quad$ Laplace transform of function $p(t)$

$S(x)=\mu(x) \mathrm{e}^{\left[-\int_{0}^{x} \mu(x) d x\right]}$
$S_{1}(x)=\mu_{1}(x) \mathrm{e}^{\left[-\int_{0}^{x} \mu_{1}(x) d x\right]}$
$S_{2}(y)=\mu_{2}(y) \mathrm{e}^{\left[-\int_{0}^{y} \mu_{2}(y) d y\right]}$
$S_{3}(z)=\mu_{3}(z) \mathrm{e}^{\left[-\int_{0}^{z} \mu_{3}(z) d z\right]}$

\section{State-Specification}

The following states of the system are common for both models:

$S_{0} / S_{1} \quad$ The new unit is operative within/ beyond warranty.

$S_{2} / S_{4} \quad$ The new unit is in failed state within/ beyond warranty.

$S_{3} \quad$ The new unit is under PM beyond warranty.

The remaining states for model-2 are:

$S_{5} \quad$ The degraded unit is operative beyond warranty.

$S_{6} \quad$ The failed degraded unit is under replacement beyond warranty.

\section{Formulation of mathematical model-1}

Using the probabilistic arguments and limiting transitions, we have the following difference-differential equations:

$$
\left[\frac{d}{d t}+\lambda+\alpha\right] p_{0}(t)=\int_{0}^{\infty} \mu(x) p_{2}(x, t) d x
$$

$\left[\frac{d}{d t}+\lambda_{1}+\lambda_{m}\right] p_{1}(t)=\alpha p_{0}(t)+\int_{0}^{\infty} \mu_{1}(x) p_{4}(x, t) d x+\int_{0}^{\infty} \mu_{2}(y) p_{3}(y, t) d y$

$$
\begin{aligned}
& {\left[\frac{\partial}{\partial t}+\frac{\partial}{\partial x}+\mu(x)\right] p_{2}(x, t)=0} \\
& {\left[\frac{\partial}{\partial t}+\frac{\partial}{\partial y}+\mu_{2}(y)\right] p_{3}(y, t)=0}
\end{aligned}
$$

$$
\left[\frac{\partial}{\partial t}+\frac{\partial}{\partial x}+\mu_{1}(x)\right] p_{4}(x, t)=0
$$

\section{Boundary Conditions}

$$
\begin{aligned}
& p_{2}(0, t)=\lambda p_{0}(t) \\
& p_{3}(0, t)=\lambda_{m} p_{1}(t)
\end{aligned}
$$




$$
p_{4}(0, t)=\lambda_{1} p_{1}(t)
$$

\section{Initial conditions}

$p_{i}(0)=1 ;$ when $i=0$

$$
p_{i}(0)=0 \text {; when } i \neq 0
$$

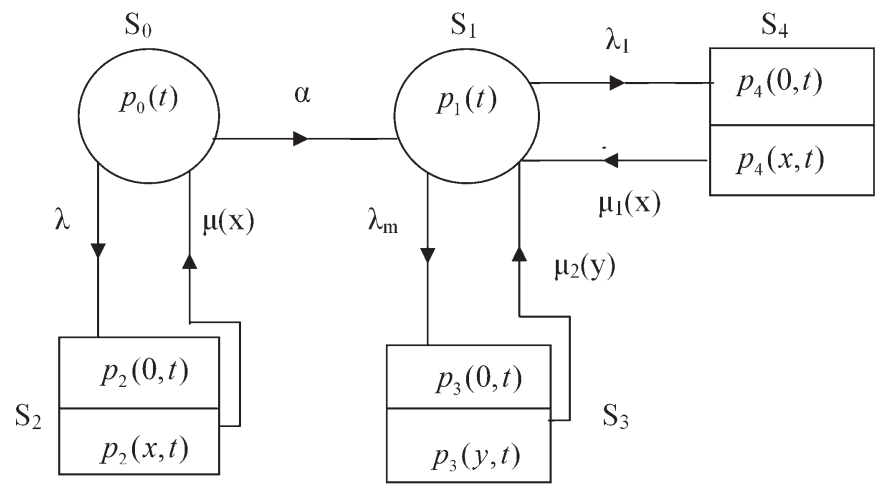

Fig. 1. State transition diagram of the model-1

\section{Analysis for model-1}

\subsection{Solution of the equations}

Taking Laplace transforms of equations (1)-(8) and using (9), we obtain:

$$
[s+\lambda+\alpha] p_{0}(s)=1+\int_{0}^{\infty} \mu(x) p_{2}(x, s) d x
$$

$\left[s+\lambda_{1}+\lambda_{m}\right] p_{1}(s)=\alpha p_{0}(s)+\int_{0}^{\infty} \mu_{1}(x) p_{4}(x, s) d x+\int_{0}^{\infty} \mu_{2}(y) p_{3}(y, s) d y$

$$
\begin{gathered}
{\left[\frac{\partial}{\partial x}+s+\mu(x)\right] p_{2}(x, s)=0} \\
{\left[\frac{\partial}{\partial y}+s+\mu_{2}(y)\right] p_{3}(y, s)} \\
{\left[\frac{\partial}{\partial x}+s+\mu_{1}(x)\right] p_{4}(x, s)=0} \\
p_{2}(0, s)=\lambda p_{0}(s) \\
p_{3}(0, s)=\lambda_{m} p_{1}(s) \\
p_{4}(0, s)=\lambda_{1} p_{1}(s)
\end{gathered}
$$

Taking integration of equations (12), (13) and (14), we get the following equations:

$$
p_{2}(x, t)=p_{2}(0, t) \mathrm{e}^{\left[-s x-\int_{0}^{x} \mu(x) d x\right]}
$$

$$
p_{3}(y, t)=p_{3}(0, t) \mathrm{e}^{\left[-s y-\int_{0}^{y} \mu_{2}(y) d y\right]}
$$

and

$$
p_{4}(x, t)=p_{4}(0, t) \mathrm{e}^{\left[-s x-\int_{0}^{x} \mu_{1}(x) d x\right]}
$$

Using equations (15) and (18), equation (10) yields:

$$
\begin{gathered}
{[s+\lambda+\alpha] p_{0}(s)=1+p_{2}(0, t) \int_{0}^{\infty} \mu(x) \mathrm{e}^{\left[-s x-\int_{0}^{x} \mu(x) d x\right]} d x=1=\lambda p_{0}(s) S(s)} \\
p_{0}(s)=\frac{1}{T(s)}
\end{gathered}
$$

where $T(s)=s+\alpha+\lambda(1-S(s))$

Using equations (16), (17), (19) and (20), equation (11) yields:

$\left[s+\lambda_{1}+\lambda_{m}\right] p_{1}(s)=\alpha p_{0}(s)+p_{4}(0, t) \int_{0}^{\infty} \mu_{1}(x) \mathrm{e}^{\left[-s x-\int_{0}^{x} \mu_{1}(x) d x\right]} d x+p_{3}(0, t) \int_{0}^{\infty} \mu_{2}(y) \mathrm{e}^{\left[-s y-\int_{0}^{y} \mu_{2}(y) d y\right]} d y$ $=\alpha p_{0}(s)+\lambda_{1} p_{1}(s) S_{1}(s)+\lambda_{m} p_{1}(s) S_{2}(s)$

$$
p_{1}(s)=\frac{A(s)}{T(s)}
$$

Where $A(s)=\frac{\alpha}{\left(s+\lambda_{1}-\lambda_{1} S_{1}(s)-\lambda_{m} S_{2}(s)\right)}$

Now, the Laplace transform of the probability that the system is in the failed state is given by:

$$
\begin{gathered}
p_{2}(s)=\int_{0}^{\infty} p_{2}(s, x) d x=\lambda p_{0}(s) \frac{(1-S(s))}{s} \\
p_{2}(s)=\frac{\lambda B(s)}{T(s)}
\end{gathered}
$$

Where $B(s)=\frac{(1-S(s))}{s}$

Similarly, $p_{3}(s)=\int_{0}^{\infty} p_{3}(s, y) d y=\lambda_{m} p_{1}(s) \frac{\left(1-S_{2}(s)\right)}{s}$

$$
p_{3}(s)=\frac{\left(\lambda_{m} A(s) C(s)\right)}{T(s)}
$$

Where $C(s)=\frac{\left(1-S_{2}(s)\right)}{s}$

Similarly, $p_{4}(s)=\int_{0}^{\infty} p_{4}(s, x) d x=\lambda_{1} p_{1}(s) \frac{\left(1-S_{1}(s)\right)}{s}$ 


$$
p_{4}(s)=\frac{\left(\lambda_{1} A(s) D(s)\right)}{T(s)}
$$

where $D(s)=\frac{\left(1-S_{1}(s)\right)}{s}$

It is worth noticing that:

$$
p_{0}(s)+p_{1}(s)+p_{2}(s)+p_{3}(s)+p_{4}(s)=\frac{1}{s}
$$

\subsection{Evaluation of Laplace Transforms of Up and Down state probabilities}

The Laplace transforms of the probabilities that the system is in Up State $\left(p_{u p}(t)\right)$ (i.e. Good State) and Down State $\left(p_{\text {down }}(t)\right)$ (i.e. Failed State) at time $t$ are as follows:

$$
\begin{gathered}
p_{u p}(s)=p_{0}(s)+p_{1}(s) \\
p_{u p}(s)=\frac{(1+A(s))}{T(s)} \\
p_{\text {down }}(s)=p_{2}(s)+p_{3}(s)+p_{4}(s) \\
p_{\text {down }}(s)=\frac{\left(\lambda B(s)+\lambda_{m} A(s) C(s)+\lambda_{1} A(s) D(s)\right)}{T(s)}
\end{gathered}
$$

\subsection{Steady-State behavior of the system}

Using Abel's Lemma ([6] \& [9]) i.e.

$\lim _{t \rightarrow \infty} F(t)=\lim _{s \rightarrow 0} s F(s)=F$ in equations (32) and (33), Provided the limit on the right hand side exists, the following time independent probabilities have been obtained:

$$
\begin{gathered}
P_{u p}=\frac{1}{\left(1-\lambda_{1} S_{1}^{\prime}(0)-\lambda_{m} S_{2}^{\prime}(0)\right)} \\
P_{d o w n}=\frac{-\lambda_{1} S_{1}^{\prime}(0)-\lambda_{m} S_{2}^{\prime}(0)}{\left(1-\lambda_{1} S_{1}^{\prime}(0)-\lambda_{m} S_{2}^{\prime}(0)\right)}
\end{gathered}
$$

\subsection{Reliability of the system $(R(t))$} are:

The differential-difference equations for reliability of the system

$$
\begin{gathered}
{\left[\frac{d}{d t}+\lambda+\alpha\right] p_{0}(t)=0} \\
{\left[\frac{d}{d t}+\lambda_{1}+\lambda_{m}\right] p_{1}(t)=\alpha p_{0}(t)}
\end{gathered}
$$

Taking Laplace transform of equations (36) and (37), using (9), we get:

$$
[s+\lambda+\alpha] p_{0}(s)=1
$$

$$
\left[s+\lambda_{1}+\lambda_{m}\right] p_{1}(s)=\alpha p_{0}(s)
$$

The solution can be written as:

$$
p_{0}(s)=\frac{1}{(s+\alpha+\lambda)}
$$

$$
=\frac{1}{(s+\alpha+\lambda)}+\frac{\alpha}{(s+\alpha+\lambda)\left(s+\lambda_{1}+\lambda_{m}\right)}
$$

Taking inverse Laplace transform, we get:

$$
R(t)=e^{-(\lambda+\alpha) t}\left[\frac{\left(\lambda-\lambda_{1}-\lambda_{m}\right)}{\left(\lambda-\lambda_{1}-\lambda_{m}+\alpha\right)}\right]+e^{-\left(\lambda_{1}+\lambda_{m}\right) t}\left[\frac{\alpha}{\left(\lambda-\lambda_{1}-\lambda_{m}+\alpha\right)}\right]
$$

\subsection{Mean time to system failure (MTSF)}

$$
\begin{gathered}
\text { MTSF }=\int_{0}^{\infty} R(t) d t \\
M T S F=\int_{0}^{\infty}\left\{e^{-(\lambda+\alpha) t}\left[\frac{\left(\lambda-\lambda_{1}-\lambda_{m}\right)}{\left(\lambda-\lambda_{1}-\lambda_{m}+\alpha\right)}\right]+e^{-\left(\lambda_{1}+\lambda_{m}\right) t}\left[\frac{\alpha}{\left(\lambda-\lambda_{1}-\lambda_{m}+\alpha\right)}\right]\right\} d t \\
M T S F=\left[\frac{\left(\lambda-\lambda_{1}-\lambda_{m}\right)}{\left(\lambda-\lambda_{1}-\lambda_{m}+\alpha\right)(\lambda+\alpha)}\right]+\left[\frac{\alpha}{\left(\lambda-\lambda_{1}-\lambda_{m}+\alpha\right)\left(\lambda_{1}+\lambda_{m}\right)}\right]
\end{gathered}
$$

\section{Formulation of mathematical model-2}

Equations (1), (3), (4) and (5) defined in model-1 are same for model- 2 and remaining equations for model- 2 are:

$$
\left[\frac{d}{d t}+\lambda_{1}+\lambda_{m}\right] p_{1}(t)=\alpha p_{0}(t)+\int_{0}^{\infty} \mu_{3}(z) p_{6}(z, t) d x+\int_{0}^{\infty} \mu_{2}(y) p_{3}(y, t) d y
$$

$$
\begin{gathered}
{\left[\frac{d}{d t}+\lambda_{2}\right] p_{5}(t)=\int_{0}^{\infty} \mu_{1}(x) p_{4}(x, t) d x} \\
{\left[\frac{\partial}{\partial t}+\frac{\partial}{\partial z}+\mu_{3}(z)\right] p_{6}(z, t)=0}
\end{gathered}
$$

\section{Boundary Conditions}

Boundary $p_{2}(0, t), p_{3}(0, t)$ and $p_{4}(0, t)$ are same as defined in model-1 and remaining is:

$$
p_{6}(0, t)=\lambda_{2} p_{5}(t)
$$




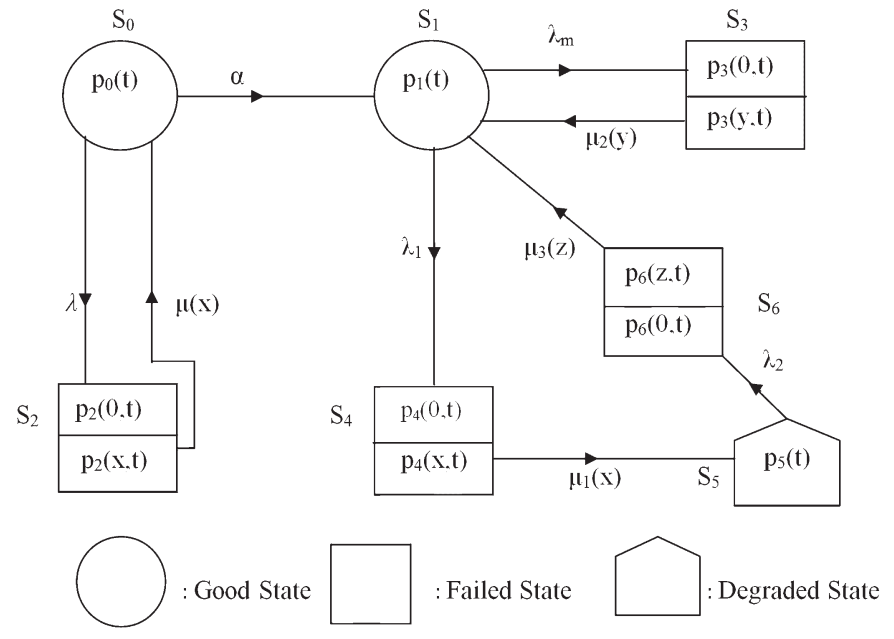

Fig. 2. Transition diagram of the model-2

\section{Analysis for model-2}

\subsection{Solution of the equations}

Proceeding in similar way as in model-1, the expressions for $p_{0}(s), p_{2}(s), p_{3}(s)$ and $p_{4}(s)$ are same as defined in model-1 and remaining expressions are:

$$
p_{1}(s)=\frac{A(s)}{T(s)}
$$

Where, $A(s)=\frac{\alpha\left(s+\lambda_{2}\right)}{\left(s+\lambda_{1}+\lambda_{m}\right)\left(s+\lambda_{2}\right)-\lambda_{m} S_{2}(s)\left(s+\lambda_{2}\right)-\lambda_{1} S_{1}(s) S_{3}(s)}$

And $T(s)$ is same as defined in model-1.

$$
p_{5}(s)=\frac{\lambda_{1} A(s) S_{1}(s)}{T(s)\left(s+\lambda_{2}\right)}
$$

Now,

$$
\begin{gathered}
p_{6}(s)=\int_{0}^{\infty} p_{6}(s, z) d z=\lambda_{2} p_{5}(s) \frac{\left(1-S_{3}(s)\right)}{s} \\
p_{6}(s)=\frac{\left(\lambda_{2} \lambda_{1} A(s) S_{1}(s) E(s)\right)}{T(s)\left(s+\lambda_{2}\right)}
\end{gathered}
$$

where $\quad E(s)=\frac{\left(1-S_{3}(s)\right)}{s}$

It is worth noticing that:

$$
p_{0}(s)+p_{1}(s)+p_{2}(s)+p_{3}(s)+p_{4}(s)+p_{5}(s)+p_{6}(s)=\frac{1}{s}
$$

$$
\begin{gathered}
p_{u p}(s)=p_{0}(s)+p_{1}(s)+p_{5}(s) \\
p_{\text {up }}(s)=\frac{\left(1+A(s)+\frac{\lambda_{1} S_{1}(s) A(s)}{\left(s+\lambda_{2}\right)}\right)}{T(s)} \\
p_{\text {down }}(s)=p_{2}(s)+p_{3}(s)+p_{4}(s)+p_{6}(s) \\
p_{\text {down }}(s)=\frac{\left(\lambda B(s)+\lambda_{m} C(s) A(s)+\lambda_{1} A(s) D(s)+\left(\frac{\lambda_{1} \lambda_{2} S_{1}(s) A(s) E(s)}{\left(s+\lambda_{2}\right)}\right)\right)}{T(s)}
\end{gathered}
$$

7.3 Steady-State behavior of the system

$$
p_{u p}=\frac{\left(\lambda_{1}+\lambda_{2}\right)}{\left(\lambda_{1}+\lambda_{2}-\lambda_{2} \lambda_{m} S_{2}^{\prime}(0)-\lambda_{1} \lambda_{2} S_{1}^{\prime}(0)-\lambda_{1} \lambda_{2} S_{3}^{\prime}(0)\right)}
$$

$$
p_{\text {down }}=\frac{-\lambda_{2} \lambda_{m} S_{2}^{\prime}(0)-\lambda_{1} \lambda_{2} S_{1}^{\prime}(0)-\lambda_{1} \lambda_{2} S_{3}^{\prime}(0)}{\left(\lambda_{1}+\lambda_{2}-\lambda_{2} \lambda_{m} S_{2}^{\prime}(0)-\lambda_{1} \lambda_{2} S_{1}^{\prime}(0)-\lambda_{1} \lambda_{2} S_{3}^{\prime}(0)\right)}
$$

\subsection{Reliability and mean time to system failure (MTSF)} el-1.

Reliability and MTSF of this model is same as that of the mod-

\section{Special cases}

\subsection{Availability of the system for model-1}

When repair and PM times follow exponential distribution i.e.

$$
S(s)=\frac{\mu}{(s+\mu)}, S_{1}(s)=\frac{\mu_{1}}{\left(s+\mu_{1}\right)} \text { and } S_{2}(s)=\frac{\mu_{2}}{\left(s+\mu_{2}\right)}
$$

where $\mu$ and $\mu_{1}$ are constant repair rates and $\mu_{2}$ is constant PM rate. Putting these values in equations (21)-(24), we get:

$$
p_{0}(s)=\frac{1}{I(s)}
$$

Where $I(s)=\frac{\left(s^{2}+s(\mu+\lambda+\alpha)+\alpha \mu\right)}{(s+\mu)}$

$$
p_{1}(s)=\frac{F(s)}{I(s)}
$$

Where

$F(s)=\frac{\alpha\left(s+\mu_{1}\right)\left(s+\mu_{2}\right)}{\left(s+\lambda_{1}+\lambda_{m}\right)\left(s+\mu_{1}\right)\left(s+\mu_{2}\right)-\lambda_{1} \mu_{1}\left(s+\mu_{2}\right)-\lambda_{m} \mu_{2}\left(s+\mu_{1}\right)}$

\subsection{Evaluation of Laplace Transforms of Up and Down state probabilities}


$p_{u p}(s)=p_{0}(s)+p_{1}(s)$

$\frac{\left(s^{3}+s^{2}\left(\lambda_{1}+\lambda_{m}+\alpha+\mu_{1}+\mu_{2}\right)+s\left(\lambda_{1} \mu_{2}+\mu_{1} \mu_{2}+\alpha \mu_{2}+\lambda_{m} \mu_{1}+\alpha \mu_{1}\right)+\alpha \mu_{1} \mu_{2}\right)\left(s+\mu_{1}\right)}{s\left(s^{2}+s(\lambda+\alpha+\mu)+\alpha \mu\right)\left(s^{2}+s\left(\lambda_{1}+\lambda_{m}+\mu_{1}+\mu_{2}\right)+\left(\lambda_{1} \mu_{2}+\mu_{1} \mu_{2}+\lambda_{m} \mu_{1}\right)\right)}$

Taking inverse Laplace transform of equation (62), we get:

$$
\begin{aligned}
p_{u p}(t) & =\frac{c_{0} \mu}{z_{1} z_{2} z_{3} z_{4}}+\left\{\frac{\left(z_{1}^{3}+c_{2} z_{1}^{2}+c_{1} z_{1}+c_{0}\right)\left(z_{1}+\mu\right)}{z_{1}\left(z_{1}-z_{2}\right)\left(z_{1}-z_{3}\right)\left(z_{1}-z_{4}\right)}\right\} \exp \left(z_{1} t\right) \\
& -\left\{\frac{\left(z_{2}^{3}+c_{2} z_{2}^{2}+c_{1} z_{2}+c_{0}\right)\left(z_{2}+\mu\right)}{z_{2}\left(z_{1}-z_{2}\right)\left(z_{2}-z_{3}\right)\left(z_{2}-z_{4}\right)}\right\} \exp \left(z_{2} t\right) \\
& +\left\{\frac{\left(z_{3}^{3}+c_{2} z_{3}{ }^{2}+c_{1} z_{3}+c_{0}\right)\left(z_{3}+\mu\right)}{z_{3}\left(z_{1}-z_{3}\right)\left(z_{2}-z_{3}\right)\left(z_{3}-z_{4}\right)}\right\} \exp \left(z_{3} t\right) \\
& -\left\{\frac{\left(z_{4}{ }^{3}+c_{2} z_{4}{ }^{2}+c_{1} z_{4}+c_{0}\right)\left(z_{4}+\mu\right)}{z_{4}\left(z_{4}-z_{1}\right)\left(z_{4}-z_{2}\right)\left(z_{3}-z_{4}\right)}\right\} \exp \left(z_{4} t\right)
\end{aligned}
$$

Where: $c_{2}=\left(\lambda_{1}+\lambda_{m}+\alpha+\mu_{1}+\mu_{2}\right), c_{1}=$

$$
=\left(\lambda_{1} \mu_{2}+\mu_{1} \mu_{2}+\alpha \mu_{2}+\lambda_{m} \mu_{1}+\alpha \mu_{1}\right), c_{0}=\alpha \mu_{1} \mu_{2}
$$

and $z_{1}, z_{2}$ are two roots of the equation $\left(s^{2}+s(\lambda+\alpha+\mu)+\alpha \mu\right)=0$ and $z_{3}, z_{4}$ are two roots of the equation $\left(s^{2}+s\left(\lambda_{1}+\lambda_{m}+\mu_{1}+\mu_{2}\right)+\left(\lambda_{1} \mu_{2}+\mu_{1} \mu_{2}+\lambda_{m} \mu_{1}\right)\right)=0$

\subsection{Availability of the system for model-2}

Proceeding in similar way as in model-1, the expressions for $p_{0}(s)$ is same as that of defined in equation (58) for model-1and remaining expressions are:

$$
p_{1}(s)=\frac{J(s)}{I(s)}
$$

Where:

$J(s)=\frac{\alpha\left(s+\mu_{1}\right)\left(s+\mu_{2}\right)\left(s+\mu_{3}\right)\left(s+\lambda_{2}\right)}{\left(s+\lambda_{1}+\lambda_{m}\right)\left(s+\mu_{1}\right)\left(s+\mu_{2}\right)\left(s+\mu_{3}\right)-\mu_{2} \lambda_{m}\left(s+\mu_{1}\right)\left(s+\mu_{3}\right)\left(s+\lambda_{2}\right)-\lambda_{1} \lambda_{2} \mu_{1} \mu_{3}\left(s+\mu_{2}\right)}$

And $I(s)$ is same as defined in equation (59):

$$
p_{5}(s)=\frac{J(s) K(s)}{I(s)}
$$

Where $K(s)=\frac{\mu_{1} \lambda_{1}}{\left(s+\mu_{1}\right)\left(s+\lambda_{2}\right)}$

$$
\begin{gathered}
p_{u p}(s)=p_{0}(s)+p_{1}(s)+p_{5}(s) \\
=\frac{\left(s^{5}+b_{4} s^{4}+b_{3} s^{3}+b_{2} s^{2}+b_{1} s+b_{0}\right)(s+\mu)}{s\left(s^{2}+s(\lambda+\alpha+\mu)+\alpha \mu\right)\left(s^{4}+a_{3} s^{3}+a_{2} s^{2}+a_{1} s+a_{0}\right)}
\end{gathered}
$$

Where $b_{4}=\left(\lambda_{1}+\lambda_{2}+\lambda_{m}+\alpha+\mu_{1}+\mu_{2}+\mu_{3}\right)$,

$b_{3}=\left(\begin{array}{l}\lambda_{1} \mu_{1}+\lambda_{1} \mu_{2}+\lambda_{2} \mu_{2}+\lambda_{1} \lambda_{2}+\lambda_{2} \mu_{1}+\lambda_{2} \mu_{3}+\lambda_{1} \mu_{3}+\lambda_{m} \mu_{1}+\lambda_{m} \mu_{3} \\ +\lambda_{m} \lambda_{2}+\mu_{1} \mu_{3}+\mu_{1} \mu_{2}+\mu_{2} \mu_{3}+\alpha \mu_{1}+\alpha \mu_{2}+\alpha \mu_{3}+\alpha \lambda_{2}\end{array}\right)$

$b_{2}=\left(\lambda_{1} \mu_{1} \mu_{3}+\lambda_{2} \mu_{1} \mu_{3}+\lambda_{2} \mu_{1} \mu_{2}+\lambda_{2} \mu_{2} \mu_{3}+\lambda_{1} \mu_{1} \mu_{2}+\lambda_{1} \mu_{2} \mu_{3}+\lambda_{m} \mu_{1} \mu_{3}+\lambda_{1} \lambda_{2} \mu_{1}+\lambda_{1} \lambda_{2} \mu_{3}+\lambda_{1} \lambda_{2} \mu_{2}\right)$ $b_{2}=\left(\begin{array}{l}\lambda_{1} \mu_{1} \mu_{3}+\lambda_{2} \mu_{1} \mu_{2}+\lambda_{2} \mu_{1} \mu_{2}+\lambda_{2} \mu_{2} \mu_{3}+\lambda_{1} \mu_{1} \mu_{2}+\lambda_{1} \mu_{2} \mu_{3}+\lambda_{m} \mu_{1} \mu_{3}+\lambda_{1} \lambda_{2} \mu_{1}+\lambda_{1} \lambda_{2} \mu_{3}+\lambda_{1} \lambda_{2} \mu_{2} \\ +\lambda_{1} \mu_{3} \mu_{3}+\alpha \mu_{3} \mu_{2}+\alpha \lambda_{2} \mu_{1}+\alpha \lambda_{2} \mu_{3}+\alpha \lambda_{2} \mu_{2}+\alpha \lambda_{1} \mu_{1}\end{array}\right)$

$b_{1}=\left(\begin{array}{c}\lambda_{1} \mu_{1} \mu_{2} \mu_{3}+\lambda_{1} \lambda_{2} \mu_{1} \mu_{2}+\lambda_{1} \lambda_{2} \mu_{3} \mu_{2}+\lambda_{m} \lambda_{2} \mu_{1} \mu_{3}+\alpha \lambda_{2} \mu_{1} \mu_{3} \\ +\alpha \lambda_{2} \mu_{1} \mu_{2}+\alpha \lambda_{2} \mu_{2} \mu_{3}+\alpha \lambda_{1} \mu_{1} \mu_{3}+\alpha \lambda_{1} \mu_{1} \mu_{2}+\alpha \mu_{1} \mu_{2} \mu_{3}\end{array}\right)$

$b_{0}=\alpha \lambda_{1} \mu_{1} \mu_{2} \mu_{3}+\alpha \lambda_{2} \mu_{1} \mu_{2} \mu_{3}$,

$a_{3}=\left(\lambda_{1}+\lambda_{2}+\lambda_{m}+\mu_{1}+\mu_{2}+\mu_{3}\right)$

$a_{2}=\left(\begin{array}{l}\lambda_{1} \mu_{1}+\lambda_{1} \mu_{3}+\lambda_{1} \lambda_{2}+\lambda_{2} \mu_{1}+\lambda_{2} \mu_{2}+\lambda_{1} \mu_{2}+\lambda_{m} \mu_{1} \\ +\lambda_{m} \mu_{3}+\lambda_{2} \mu_{3}+\lambda_{m} \lambda_{2}+\mu_{1} \mu_{3}+\mu_{1} \mu_{2}+\mu_{2} \mu_{3}\end{array}\right)$

$a_{1}=\left(\begin{array}{l}\lambda_{1} \mu_{1} \mu_{3}+\lambda_{2} \mu_{1} \mu_{3}+\lambda_{2} \mu_{1} \mu_{2}+\lambda_{2} \mu_{2} \mu_{3}+\lambda_{1} \mu_{1} \mu_{2}+\lambda_{1} \mu_{2} \mu_{3}+\lambda_{1} \lambda_{2} \mu_{1} \\ +\lambda_{1} \lambda_{2} \mu_{3}+\lambda_{1} \lambda_{2} \mu_{2}+\lambda_{m} \mu_{1} \mu_{3}++\lambda_{m} \lambda_{2} \mu_{1}+\lambda_{m} \lambda_{2} \mu_{3}+\mu_{1} \mu_{2} \mu_{3}\end{array}\right)$

and $a_{0}=\left(\lambda_{1} \mu_{1} \mu_{2} \mu_{3}+\lambda_{1} \lambda_{2} \mu_{1} \mu_{2}+\lambda_{1} \lambda_{2} \mu_{3} \mu_{2}+\lambda_{m} \lambda_{2} \mu_{1} \mu_{3}\right)$

Taking inverse Laplace transform of equation (68), we get:

$p_{u p}(t)=\frac{b_{0} \mu}{z_{1} z_{2} z_{3} z_{4} z_{5} z_{6}}+\left\{\frac{\left(z_{1}^{5}+b_{4} z_{1}^{4}+b_{3} z_{1}^{3}+b_{2} z_{1}^{2}+b_{1} z_{1}+b_{0}\right)\left(z_{1}+\mu\right)}{z_{1}\left(z_{1}-z_{2}\right)\left(z_{1}-z_{3}\right)\left(z_{1}-z_{4}\right)\left(z_{1}-z_{5}\right)\left(z_{1}-z_{6}\right)}\right\} \exp \left(z_{1} t\right)$

$+\left\{\frac{\left(z_{2}^{5}+b_{4} z_{2}^{4}+b_{3} z_{2}^{3}+b_{2} z_{2}^{2}+b_{1} z_{2}+b_{0}\right)\left(z_{2}+\mu\right)}{z_{2}\left(z_{2}-z_{1}\right)\left(z_{2}-z_{3}\right)\left(z_{2}-z_{4}\right)\left(z_{2}-z_{5}\right)\left(z_{2}-z_{6}\right)}\right\} \exp \left(z_{2} t\right)$

$+\left\{\frac{\left(z_{3}^{5}+b_{4} z_{3}^{4}+b_{3} z_{3}^{3}+b_{2} z_{3}^{2}+b_{1} z_{3}+b_{0}\right)\left(z_{3}+\mu\right)}{z_{3}\left(z_{3}-z_{1}\right)\left(z_{3}-z_{2}\right)\left(z_{3}-z_{4}\right)\left(z_{3}-z_{5}\right)\left(z_{3}-z_{6}\right)}\right\} \exp \left(z_{3} t\right)$

$+\left\{\frac{\left(z_{4}{ }^{5}+b_{4} z_{4}{ }^{4}+b_{3} z_{4}{ }^{3}+b_{2} z_{4}{ }^{2}+b_{1} z_{4}+b_{0}\right)\left(z_{4}+\mu\right)}{z_{4}\left(z_{4}-z_{1}\right)\left(z_{4}-z_{2}\right)\left(z_{4}-z_{3}\right)\left(z_{4}-z_{5}\right)\left(z_{4}-z_{6}\right)}\right\} \exp \left(z_{4} t\right)$

$+\left\{\frac{\left(z_{5}^{5}+b_{4} z_{5}^{4}+b_{3} z_{5}^{3}+b_{2} z_{5}^{2}+b_{1} z_{5}+b_{0}\right)\left(z_{5}+\mu\right)}{z_{5}\left(z_{5}-z_{1}\right)\left(z_{5}-z_{2}\right)\left(z_{5}-z_{3}\right)\left(z_{5}-z_{4}\right)\left(z_{5}-z_{6}\right)}\right\} \exp \left(z_{5} t\right)$

$+\left\{\frac{\left(z_{6}^{5}+b_{4} z_{6}{ }^{4}+b_{3} z_{6}{ }^{3}+b_{2} z_{6}{ }^{2}+b_{1} z_{6}+b_{0}\right)\left(z_{6}+\mu\right)}{z_{6}\left(z_{6}-z_{1}\right)\left(z_{6}-z_{2}\right)\left(z_{6}-z_{3}\right)\left(z_{6}-z_{4}\right)\left(z_{6}-z_{5}\right)}\right\} \exp \left(z_{6} t\right)$

$z_{1}$ and $z_{2}$ are roots of the equation $\left(s^{2}+s(\lambda+\alpha+\mu)+\alpha \mu\right)=0$ and $z_{3}, z_{4}, z_{5}$ and $z_{6}$ are roots of the equation $\left(s^{4}+a_{3} s^{3}+a_{2} s^{2}+a_{1} s+a_{0}\right)=0$.

\section{Profit analysis of the User}

Suppose that the warranty period of the system is $(0, w)$. Since the repairman is always available with the system, therefore beyond warranty period, it remains busy during the interval $(w, t)$. Let $\mathrm{K}_{1}$ be the revenue per unit time and $\mathrm{K}_{2}$ be the repair cost per unit time, then the expected profits $\mathrm{H}_{1}(\mathrm{t})$ and $\mathrm{H}_{2}(\mathrm{t})$ for model-1 and 2 during the interval $(0, t)$ are given by

\section{For model-1}

Using equation (63), we get the expected profit $\mathrm{H}_{1}(\mathrm{t})$ as: 


$$
\begin{aligned}
& H_{1}(t)=K_{1} \int_{0}^{t} p_{u p}(t) d t-K_{2}(t-w) \\
& H_{1}(t)=K_{1}\left\{\begin{array}{l}
\frac{c_{0} \mu t}{z_{1} z_{2} z_{3} z_{4}}-\left\{\frac{\left(z_{1}^{3}+c_{2} z_{1}^{2}+c_{1} z_{1}+c_{0}\right)\left(z_{1}+\mu\right)}{z_{1}^{2}\left(z_{1}-z_{2}\right)\left(z_{1}-z_{3}\right)\left(z_{1}-z_{4}\right)}\right\}\left(1-e^{z_{1} t}\right) \\
\left\{\frac{\left(z_{2}^{3}+c_{2} z_{2}^{2}+c_{1} z_{2}+c_{0}\right)\left(z_{2}+\mu\right)}{z_{2}^{2}\left(z_{1}-z_{2}\right)\left(z_{2}-z_{3}\right)\left(z_{2}-z_{4}\right)}\right\}\left(1-e^{z_{2} t}\right) \\
-\left\{\frac{\left(z_{3}^{3}+c_{2} z_{3}^{2}+c_{1} z_{3}+c_{0}\right)\left(z_{3}+\mu\right)}{z_{3}^{2}\left(z_{1}-z_{3}\right)\left(z_{2}-z_{3}\right)\left(z_{3}-z_{4}\right)}\right\}\left(1-e^{z_{3} t}\right) \\
+\left\{\frac{\left(z_{4}^{3}+c_{2} z_{4}^{2}+c_{1} z_{4}+c_{0}\right)\left(z_{4}+\mu\right)}{z_{4}^{2}\left(z_{4}-z_{1}\right)\left(z_{4}-z_{2}\right)\left(z_{3}-z_{4}\right)}\right\}\left(1-e^{z_{4} t}\right)
\end{array}\right\}-K_{2}(t-w)
\end{aligned}
$$

\section{For model-2}

Using equation (69), we get the expected profit $\mathrm{H}_{2}(\mathrm{t})$ as:

$$
\left.\begin{array}{c}
H_{2}(t)=K_{1} \int_{0}^{t} p_{u p}(t) d t-K_{2}(t-w) \\
+\frac{b_{0} \mu t}{z_{1} z_{2} z_{3} z_{4} z_{5} z_{6}}+\left\{\frac{\left(z_{1}^{5}+b_{4} z_{1}^{4}+b_{3} z_{1}^{3}+b_{2} z_{1}^{2}+b_{1} z_{1}+b_{0}\right)\left(z_{1}+\mu\right)}{z_{1}^{2}\left(z_{1}-z_{2}\right)\left(z_{1}-z_{3}\right)\left(z_{1}-z_{4}\right)\left(z_{1}-z_{5}\right)\left(z_{1}-z_{6}\right)}\right\}\left(e^{z_{1} t}-1\right) \\
+\left\{\frac{\left(z_{2}^{5}+b_{4} z_{2}^{4}+b_{3} z_{2}^{3}+b_{2} z_{2}^{2}+b_{1} z_{2}+b_{0}\right)\left(z_{2}+\mu\right)}{z_{2}^{2}\left(z_{2}-z_{1}\right)\left(z_{2}-z_{3}\right)\left(z_{2}-z_{4}\right)\left(z_{2}-z_{5}\right)\left(z_{2}-z_{6}\right)}\right\}\left(e^{z_{2} t}-1\right) \\
+\left\{\frac{\left(z_{3}^{5}+b_{4} z_{3}^{4}+b_{3} z_{3}^{3}+b_{2} z_{3}^{2}+b_{1} z_{3}+b_{0}\right)\left(z_{3}+\mu\right)}{z_{3}^{2}\left(z_{3}-z_{1}\right)\left(z_{3}-z_{2}\right)\left(z_{3}-z_{4}\right)\left(z_{3}-z_{5}\right)\left(z_{3}-z_{6}\right)}\right\}\left(e^{z_{3} t}-1\right) \\
+\left\{\frac{\left(z_{4}^{5}+b_{4} z_{4}^{4}+b_{3} z_{4}^{3}+b_{2} z_{4}{ }^{2}+b_{1} z_{4}+b_{0}\right)\left(z_{4}+\mu\right)}{z_{4}^{2}\left(z_{4}-z_{1}\right)\left(z_{4}-z_{2}\right)\left(z_{4}-z_{3}\right)\left(z_{4}-z_{5}\right)\left(z_{4}-z_{6}\right)}\right\}\left(e^{z_{4} t}-1\right) \\
+\left\{\frac{\left(z_{5}^{5}+b_{4} z_{5}^{4}+b_{3} z_{5}^{3}+b_{2} z_{5}^{2}+b_{1} z_{5}+b_{0}\right)\left(z_{5}+\mu\right)}{z_{5}^{2}\left(z_{5}-z_{1}\right)\left(z_{5}-z_{2}\right)\left(z_{5}-z_{3}\right)\left(z_{5}-z_{4}\right)\left(z_{5}-z_{6}\right)}\right\}\left(e^{z_{5} t}-1\right) \\
+\left\{\frac{\left(z_{6}^{5}+b_{4} z_{6}^{4}+b_{3} z_{6}^{3}+b_{2} z_{6}^{2}+b_{1} z_{6}+b_{0}\right)\left(z_{6}+\mu\right)}{z_{6}^{2}\left(z_{6}-z_{1}\right)\left(z_{6}-z_{2}\right)\left(z_{6}-z_{3}\right)\left(z_{6}-z_{4}\right)\left(z_{6}-z_{5}\right)}\right\}\left(e^{z_{6} t}-1\right)
\end{array}\right\}
$$

\begin{tabular}{|c|c|c|c|c|c|}
\hline \multirow{2}{*}{$\begin{array}{l}\text { Time } \\
(\mathrm{t})\end{array}$} & $\begin{array}{l}\lambda_{1}=0.02 \\
\mathrm{a}=0.003 \\
\lambda_{\mathrm{m}}=0.04\end{array}$ & $\begin{array}{l}\lambda_{1}=0.02 \\
\mathrm{a}=0.003 \\
\lambda_{\mathrm{m}}=0.04\end{array}$ & $\begin{array}{l}\lambda=0.01 \\
a=0.003 \\
\lambda_{m}=0.04\end{array}$ & $\begin{array}{l}\lambda=0.01 \\
\lambda_{1}=0.02 \\
\lambda_{\mathrm{m}}=0.04\end{array}$ & $\begin{array}{c}\lambda=0.01 \\
\lambda_{1}=0.02 \\
a=0.003\end{array}$ \\
\hline & $\begin{array}{c}\mathrm{R}(\mathrm{t}) \\
\text { (for } \lambda=0.01)\end{array}$ & $\begin{array}{c}\mathrm{R}(\mathrm{t}) \\
\text { (for } \lambda=0.02 \text { ) }\end{array}$ & $\begin{array}{c}\mathrm{R}(\mathrm{t}) \\
\left(\text { for } \lambda_{1}=0.03\right)\end{array}$ & $\begin{array}{c}\mathrm{R}(\mathrm{t}) \\
(\text { for } \mathrm{a}=0.005 \text { ) }\end{array}$ & $\begin{array}{c}\mathrm{R}(\mathrm{t}) \\
\left(\text { for } \lambda_{\mathrm{m}}=0.05\right)\end{array}$ \\
\hline 10 & 0.899114 & 0.814457 & 0.898175 & 0.895363 & 0.898175 \\
\hline 11 & 0.889088 & 0.797518 & 0.888004 & 0.884676 & 0.888004 \\
\hline 12 & 0.8791 & 0.780872 & 0.877867 & 0.873994 & 0.877867 \\
\hline 13 & 0.869154 & 0.764518 & 0.867771 & 0.863327 & 0.867771 \\
\hline 14 & 0.859254 & 0.748454 & 0.857722 & 0.852681 & 0.857722 \\
\hline 15 & 0.849405 & 0.732679 & 0.847724 & 0.842066 & 0.847724 \\
\hline 16 & 0.83961 & 0.717189 & 0.837782 & 0.831487 & 0.837782 \\
\hline 17 & 0.829873 & 0.701984 & 0.827901 & 0.820952 & 0.827901 \\
\hline
\end{tabular}

\section{Numerical analysis}

Table 1. Effect of failure rates ( $\lambda$ and $\left.\lambda_{1}\right)$, transition rate $\left(\lambda_{\mathrm{m}}\right)$ and transition rate of completion of warranty $(\alpha)$ on Reliability of the system $(R(t))$ 
Table 2. Effect of repair cost $\left(K_{2}\right), P M$ rate $\left(\mu_{2}\right)$ and transition rate by which unit goes for $P M\left(\lambda_{m}\right)$ on expected profit $\left(H_{1}(t)\right)$

\begin{tabular}{|c|c|c|c|c|}
\hline \multirow[t]{2}{*}{$\begin{array}{l}\text { Time } \\
(\mathrm{t})\end{array}$} & $\begin{array}{c}\lambda=0.01, \mu_{1}=0.1 \\
\lambda_{1}=0.02, \mu_{2}=0.3 \\
a=0.003, \mu=0.2 \\
\lambda_{m}=0.04, K_{1}=500 \\
W=3\end{array}$ & $\begin{array}{c}\lambda=0.01, \mu_{1}=0.1, \\
\lambda_{1}=0.02, \mu_{2}=0.3, \\
a=0.003, \\
\mu=0.2, \lambda_{\mathrm{m}}=0.04, \\
\mathrm{~K}_{1}=500, \mathrm{~W}=3\end{array}$ & $\begin{array}{c}\lambda=0.01, \mu_{1}=0.1 \\
\lambda_{1}=0.02, \mu=0.2 \\
a=0.003, W=3 \\
\lambda_{\mathrm{m}}=0.04, \mathrm{~K}_{1}=500 \\
\mathrm{~K}_{2}=150\end{array}$ & $\begin{array}{c}\lambda=0.01, \mu_{1}=0.1, \\
\lambda_{1}=0.02, \mu_{2}=0.3 \\
a=0.003, W=3, \\
\mu=0.2, \mathrm{~K}_{1}=500, \\
\mathrm{~K}_{2}=150\end{array}$ \\
\hline & $\begin{array}{c}\mathrm{H}_{1}(\mathrm{t}) \\
\left(\text { For } \mathrm{K}_{2}=150\right)\end{array}$ & $\begin{array}{c}\mathrm{H}_{1}(\mathrm{t}) \\
\left.\text { (For } \mathrm{K}_{2}=100\right)\end{array}$ & $\begin{array}{c}\mathrm{H}_{1}(\mathrm{t}) \\
\left(\text { For } \mu_{2}=0.4\right)\end{array}$ & $\begin{array}{c}\mathrm{H}_{1}(\mathrm{t}) \\
\left.\text { (For } \lambda_{m}=0.03\right)\end{array}$ \\
\hline 10 & 3807.814 & 4157.814 & 3808.29 & 3809.311 \\
\hline 11 & 4135.365 & 4535.365 & 4136.008 & 4137.224 \\
\hline 12 & 4462.205 & 4912.205 & 4463.044 & 4464.464 \\
\hline 13 & 4788.424 & 5288.424 & 4789.488 & 4791.118 \\
\hline 14 & 5114.094 & 5664.094 & 5115.411 & 5117.259 \\
\hline 15 & 5439.274 & 6039.274 & 5440.873 & 5442.942 \\
\hline 16 & 5764.01 & 6414.01 & 5765.921 & 5768.216 \\
\hline 17 & 6088.342 & 6788.342 & 6090.592 & 6093.117 \\
\hline
\end{tabular}

Table 3. Effect of repair cost $\left(K_{2}\right)$, , PM rate $\left(\mu_{2}\right)$, failure rate of degraded unit $\left(\lambda_{2}\right)$, transition rate by which unit goes for $P M\left(\lambda_{m}\right)$ and replacement rate of failed degraded unit $\left(\mu_{3}\right)$ on expected profit $\left(H_{2}(t)\right)$

\begin{tabular}{|c|c|c|c|c|c|c|}
\hline $\begin{array}{l}\text { Time } \\
(\mathrm{t})\end{array}$ & $\begin{array}{c}\lambda=0.01, \\
\lambda_{1}=0.02, \\
\lambda_{2}=0.03, \\
\lambda_{\mathrm{m}}=0.04, \\
a=0.003, \\
\mu=0.2, \\
\mu_{1}=0.1, \\
\mu_{2}=0.3, \\
\mu_{3}=0.4, \\
\mathrm{~W}=3, \\
\mathrm{~K}_{1}=500\end{array}$ & $\begin{array}{c}\lambda=0.01, \\
\lambda_{1}=0.02, \\
\lambda_{2}=0.03, \\
\lambda_{\mathrm{m}}=0.04, \\
a=0.003, \\
\mu=0.2, \\
\mu_{1}=0.1, \\
\mu_{2}=0.3, \\
\mu_{3}=0.4, \\
\mathrm{~W}=3, \\
\mathrm{~K}_{1}=500\end{array}$ & $\begin{array}{c}\lambda=0.01 \\
\lambda_{1}=0.02 \\
\lambda_{2}=0.03 \\
\lambda_{\mathrm{m}}=0.04 \\
a=0.003, \\
\mu=0.2 \\
\mu_{1}=0.1 \\
\mu_{3}=0.4 \\
\mathrm{~W}=3, \\
\mathrm{~K}_{1}=500 \\
\mathrm{~K}_{2}=150\end{array}$ & $\begin{array}{c}\lambda=0.01, \\
\lambda_{1}=0.02, \\
\lambda_{\mathrm{m}}=0.04, \\
a=0.003, \\
\mu=0.2, \\
\mu_{1}=0.1, \\
\mu_{1}=0.3, \\
\mu_{3}=0.4, \\
W=3, \\
\mathrm{~K}_{1}=500, \\
\mathrm{~K}_{2}=150\end{array}$ & $\begin{array}{c}\lambda=0.01, \\
\lambda_{1}=0.02, \\
\lambda_{2}=0.03, \\
a=0.003, \\
\mu=0.2, \\
\mu_{1}=0.1, \\
\mu_{2}=0.3, \\
\mu_{3}=0.4, \\
W=3, \\
\mathrm{~K}_{1}=500 \\
\mathrm{~K}_{2}=150\end{array}$ & $\begin{array}{c}\lambda=0.01, \\
\lambda_{1}=0.02, \\
\lambda_{2}=0.03, \\
\lambda_{\mathrm{m}}=0.04, \\
a=0.003, \\
\mu=0.2, \\
\mu_{1}=0.1, \\
\mu_{2}=0.3, \\
\mathrm{~W}=3, \\
\mathrm{~K}_{1}=500 \\
\mathrm{~K}_{2}=150\end{array}$ \\
\hline & $\begin{array}{c}\mathrm{H}_{2}(\mathrm{t}) \\
\left(\text { For } \mathrm{K}_{2}=150\right)\end{array}$ & $\begin{array}{c}\mathrm{H}_{2}(\mathrm{t}) \\
\left(\text { For } \mathrm{K}_{2}=100\right)\end{array}$ & $\begin{array}{c}\mathrm{H}_{2}(\mathrm{t}) \\
\left(\text { For } \mu_{2}=0.4\right)\end{array}$ & $\begin{array}{c}\mathrm{H}_{2}(\mathrm{t}) \\
\left(\text { For } \lambda_{2}=0.02\right)\end{array}$ & $\begin{array}{c}\mathrm{H}_{2}(\mathrm{t}) \\
\left(\text { For } \lambda_{\mathrm{m}}=0.03\right)\end{array}$ & $\begin{array}{c}\mathrm{H}_{2}(\mathrm{t}) \\
\left(\text { For } \mu_{3}=0.5\right)\end{array}$ \\
\hline 10 & 3804.924 & 4154.924 & 3805.578 & 3805.106 & 3806.008 & 3804.95 \\
\hline 11 & 4132.078 & 4532.078 & 4132.946 & 4132.232 & 4133.431 & 4132.119 \\
\hline 12 & 4458.548 & 4908.548 & 4459.667 & 4458.66 & 4460.201 & 4458.61 \\
\hline 13 & 4784.439 & 5284.439 & 4785.845 & 4784.488 & 4786.421 & 4784.527 \\
\hline 14 & 5109.836 & 5659.836 & 5111.57 & 5109.851 & 5112.178 & 5109.957 \\
\hline 15 & 5434.816 & 6034.816 & 5436.915 & 5434.971 & 5437.547 & 5434.976 \\
\hline 16 & 5759.44 & 6409.44 & 5761.946 & 5759.456 & 5762.59 & 5759.647 \\
\hline 17 & 6083.764 & 6783.764 & 6086.717 & 6083.809 & 6087.363 & 6084.026 \\
\hline
\end{tabular}

Table 4. Expected profit difference $\left(H_{1}(t)-H_{2}(t)\right)$

\begin{tabular}{|c|c|c|c||}
\hline \multirow{2}{*}{$\begin{array}{c}\text { Time } \\
(\mathrm{t})\end{array}$} & \multicolumn{3}{|c|}{$\lambda=0.01, \lambda_{1}=0.02, \lambda_{2}=0.03, \lambda_{\mathrm{m}}=0.04, \alpha=0.003, \mu=0.2, \mu_{1}=0.1, \mu_{2}=0.3, \mu_{3}=0.4$} \\
\cline { 2 - 4 } & $\mathrm{K}_{1}$ & $\mathrm{~W}$ & $\mathrm{H}_{1}(\mathrm{t})-\mathrm{H}_{2}(\mathrm{t})$ \\
\hline 10 & 500 & 3 & 2.890131 \\
\hline 11 & 500 & 3 & 3.287116 \\
\hline 12 & 500 & 3 & 3.657437 \\
\hline 13 & 500 & 3 & 3.985432 \\
\hline 14 & 500 & 3 & 4.258423 \\
\hline 15 & 500 & 3 & 4.457927 \\
\hline 16 & 500 & 3 & 4.570277 \\
\hline 17 & 500 & 3 & 4.577749 \\
\hline
\end{tabular}




\section{Interpretation and conclusion}

Table 1 shows that reliability for both the models is same. It is found that reliability of the system decreases with the increase of failure rates $\left(\lambda\right.$ and $\left.\lambda_{1}\right)$, transition rate by which unit goes for PM $\left(\lambda_{\mathrm{m}}\right)$ and transition rate of completion of warranty $(\alpha)$ with respect to time and for fixed values of other parameters. Tables 2 and 3 highlight the behaviour of expected profit for model-1 and 2. From table 2, it is observed that expected profit $\mathrm{H}_{1}(\mathrm{t})$ increases with the decrease of repair cost $\left(K_{2}\right)$ and transition rate by which unit goes for PM $\left(\lambda_{\mathrm{m}}\right)$ while with the increase of PM rate $\left(\mu_{2}\right)$ with respect to time. From Table 3, it is analyzed that expected profit $\mathrm{H}_{2}(\mathrm{t})$ increases with the increase of PM rate $\left(\mu_{2}\right)$ and replacement rate of failed degraded unit $\left(\mu_{3}\right)$ while with the decrease of failure rate $\left(\lambda_{2}\right)$, transition rate by which unit goes for PM $\left(\lambda_{\mathrm{m}}\right)$ and repair cost $\left(\mathrm{K}_{2}\right)$ with respect to time. Table 4 shows the expected profit difference $\left(\mathrm{H}_{1}(\mathrm{t})-\mathrm{H}_{2}(\mathrm{t})>0\right)$ which goes on increasing with respect to time. This implies that model-1 is profitable over model-2.

Hence study reveals that after getting PM beyond warranty, a system in which unit works with reduced capacity after its repair will not be economically beneficial to use.

\section{References}

1. Cox D R. The analysis of non- Markovian stochastic process by the inclusion of supplementary variables. Proc. Camb. Phil. Soc. 1955; 51: 433-441, http://dx.doi.org/10.1017/S0305004100030437.

2. Kadyan M S. Reliability and profit analysis of a single-unt system with preventive maintenance subject to maximum operation time. Eksploatacja i Niezawodnosc - Maintenance and Reliability 2013;15(2):176-181.

3. Kadyan M S, Promila, Kumar J. Reliability modeling of a single-unit system with arbitrary distribution subject to different weather condition. Int. J. Syst. Assur. Eng. Manag. 2014; 5(3):313-319, http://dx.doi.org/10.1007/s13198-013-0168-3.

4. Kumar J, Kadyan M S, Malik S C. Profit analysis of a 2-out-of-2 redundant system with single standby and degradation of the units after repair. Int. J. Syst. Assur. Eng. Manag. 2013; 4(4): 424-434, http://dx.doi.org/10.1007/s13198-012-0127-4.

5. Kumar J, Kadyan M S, Malik S C. Cost analysis of a two-unit cold standby system subject to degradation, inspection and priority. Eksploatacja i Niezawodnosc - Maintenance and Reliability 2012; 14 (4): 278-283.

6. Nianfu Yang, Dhillon B S. Stochastic analysis of a general standby system with constant human error and arbitrary system repair rates. Microelectron Reliab. 1995; 35(7): 1037-1045, http://dx.doi.org/10.1016/0026-2714(95)90862-K.

7. Nailwal B, Singh S B. Reliability and sensitivity analysis of a operating system with inspection in different weather conditions. Int. J. Reliab. Qual. Safe. Eng. 2012; 19(2):1-36, http://dx.doi.org/10.1142/S021853931250009X.

8. Ocon R P, Castro J E R, Two models for a repairable two-system with phase-type sojourn time distributions. Reliab. Eng. Syst. Safe. 2004; 84: 253-260, http://dx.doi.org/10.1016/j.ress.2003.11.006.

9. Scarf Philip A, Cavalcante Cristiano A V. Modeling quality in replacement and inspection maintenance. Int. J. Prod. Eco. 2012; 135(1): 372381, http://dx.doi.org/10.1016/j.ijpe.2011.08.011.

10. Singh V V, Singh S B, Ram M, Goel C K. Availability, MTTF and cost analysis of a system having two units in series configuration with controller. Int. J. Syst. Assur. Eng. Manag. 2013; 4(4): 341-352, http://dx.doi.org/10.1007/s13198-012-0102-0.

11. Yuan Li, Meng Xian-Yun. Reliability analysis of a warm standby repairable system with priority in use. Appl. Math. Model. 2011; 35: 42954303, http://dx.doi.org/10.1016/j.apm.2011.03.002.

\section{Ram NIWAS \\ MS KADYAN \\ Jitender KUMAR}

Department of Statistics \& O.R.

Kurukshetra University

Kurukshetra, India

E-mail: burastat0001@gmail.com,mskadian@kuk.ac.in, khatkarjitu@gmail.com 